\title{
Medizin am Fluchtweg
}

Erhard Taverna

erhard.taverna[at]saez.ch

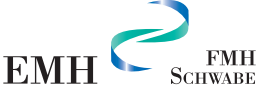

SCHWABE

Editores Medicorum Helveticorum
Zehn bis fünfzehn kommen jede Woche, Tibeter, Äthiopier, Afghanen, Eritreer, Kosovaren, Kurden, Nordafrikaner und Syrer, einzeln oder in Familien. Die Praxis von Charlotte Hartmann, FMH Allgemein-Innere Medizin, liegt weitab von politischen Epizentren, doch jedes Erdbeben macht sich früher oder später in der Sprechstunde bemerkbar. Sie kommen als Asylbewerber vom nahegelegenen Zentrum Landegg, einem ehemaligen Kur- und Seminarhotel, das von den Kantonen St. Gallen und Appenzell Ausserrhoden gemeinsam betrieben wird. 125 Asylsuchende finden dort eine Unterkunft und Betreuung, bis das Verfahren entschieden ist. Seit der Eröffnung 2010 betreut die Allgemeinärztin einmal wöchentlich Patienten, die mit dem heimeigenen Bus nach Rorschach gefahren werden, wo sie, zusammen mit ihrem Arzt-Ehemann, arbeitet. Beide bieten eine breitgefächerte Grundversorgung, inklusive Kleinchirurgie, Gynäkologie und Päd-iatrie. Eine hilfreiche Vorbereitung sei das gemeinsame Jahr in Jemen gewesen. Nach einem Kurs im Tropenmedizinischen Institut Basel bauten sie dort 1984/85 im Rahmen der Schweizerischen Katastrophenhilfe einen Sanitätsdienst auf. Dort hat die Ärztin auch das Hocharabisch gelernt, das ihr neben Kenntnissen in Italienisch, Spanisch, Französisch und Englisch gute Dienste leistet. Damit kommt sie ohne Dolmetscher aus, die nur im Zentrumsspital zur Verfügung stehen und für ihre Bedürfnisse viel zu teuer wären.

Für Frau Hartmann ist diese Patientengruppe eine willkommene Abwechslung, denn die Menschen sind im Durchschnitt jünger und ihre Probleme eine medizinische, soziale und linguistische Herausforderung. Infekte stehen im Vordergrund, vor allem Augenentzündungen und immer wieder einmal Tuberkulosefälle. Da an der Grenze praktisch keine Untersuchungen stattfinden, ist hier die Diagnostikerin besonders gefragt. An zweiter Stelle verursachen Knochen und Muskeln Beschwerden, daneben gibt es Kreislaufprobleme wie Hypertonie und immer wieder Diabetes. Psychische Symptome als Stressfolgen, wie Insomnie und Unruhe, betreffen fast die Hälfte aller Hilfesuchenden. Folteropfer mit schlecht verheilten Verletzungen sind relativ häufig. Eine Art erste posttraumatische Hilfe findet in der Praxis statt, Linderung verschaffen Sedativa, wie Lexotanil und Temesta. Für weiterführende Abklärungen und Therapien reicht die Aufenthaltsdauer in Landegg nicht. Die Krankenakten reisen, wie die Flüchtlinge mit Asylstatus, in die nächste Stadt oder das nächste Dorf zum betreuenden Arzt. Notfälle überweist Frau Hartmann an das Gemeindespital in Rorschach oder in das Kantonsspital St. Gallen, wo die Behandlung ausschliesslich nach medizinischen Kriterien, unabhängig vom ausstehen- den Entscheid des Asylverfahrens, erfolgt. Wenn eine Ausschaffung bevorsteht, wird eine offene Tuberkulose so lange behandelt, bis keine Ansteckungsgefahr mehr besteht. Eine Pflegefachfrau im Asylzentrum koordiniert die Medikamentenabgabe, sie fungiert dort als erste Triagestelle und verlängerter Arm der Praxis.

Während der ganzen Zeit sei sie nie bedroht worden. Es

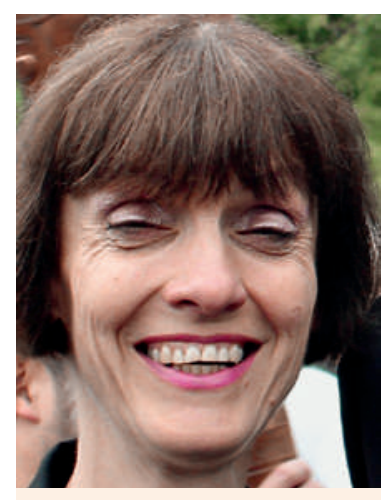

Für Charlotte Hartmann ist die Betreuung der Asylbewerber aus dem eine willkommene Bereicherung ihrer medizinischen Tätigkeit. nahegelegenen Zentrum komme vor, dass gewisse Nationalitäten besonders aggressiv und fordernd aufträten, besonders jene, die wenig Hoffnung auf eine Aufnahme hätten. Diese seien aber auch unter den Mitasylanten unbeliebt und isoliert. Auf Drogen wird nicht routinemäs-sig geprüft. Wenn schon, geht es meistens um Alkohol, besonders bei jungen Männern, die schon Jahre illegal in Italien lebten. Reibereien finden ausserhalb der Praxisräume statt, dort wo kulturell völlig unterschiedliche Menschen in engen Wohnverhältnissen miteinander auskommen müssen. Das ungewohnte Essen und die fremde Mentalität der neuen Umgebung seien ein Dauerthema. Die wöchentlichen Fälle haben das Praxisspektrum medizinisch bereichert. Viele Probleme sind bei unserer einheimischen Bevölkerung kaum zu finden, etwa ein häufig festgestellter Vitamin-D-Mangel, der zu einer erhöhten Infektanfälligkeit führt, so dass die Messung des Blutspiegels inzwischen zur Routine gehört. Es gibt auch fortgeschrittene Krankheitsstadien, zum Beispiel bei Tumoren, wie sie in unserem Gesundheitssystem nicht mehr vorkommen.

Bei Notfällen und Ferienabwesenheiten hilft ein Kollege aus Appenzell Ausserrhoden aus. Er ist für ein «Appenzeller-Drittel» der Zentrumsbewohner zuständig. Die 57-jährige Ärztin hat ausser einem vielseitigen Beruf auch stets einen Ehepartner und vier Kinder versorgt. Letztere sind alle flügge und stecken erfolgreich in einer Ausbildung. Als wäre das alles noch nicht genug, schaut Charlotte Hartmann auf ihre Uhr, denn abends ist noch eine Orchesterprobe angesagt. Sie spielt die zweite Geige bei den Musikfreunden St.Gallen. Zur Aufführung kamen im Dezember Werke von Jean Sibelius, Jonathan C. Meier und Max Reger. Ein Newsletter verrät das Programm 2013: www.musikfreunde.ch 